\title{
Moments of the product and ratio of two correlated chi-square variables
}

\author{
Anwar H. Joarder
}

Received: 2 June 2006 / Revised: 8 October 2007 / Published online: 20 November 2007

(C) The Author(s) 2007

\begin{abstract}
The exact probability density function of a bivariate chi-square distribution with two correlated components is derived. Some moments of the product and ratio of two correlated chi-square random variables have been derived. The ratio of the two correlated chi-square variables is used to compare variability. One such application is referred to. Another application is pinpointed in connection with the distribution of correlation coefficient based on a bivariate $t$ distribution.
\end{abstract}

Keywords Bivariate chi-square distribution - Moments · Product of correlated chi-square variables $\cdot$ Ratio of correlated chi-square variables

Mathematics Subject Classification (2000) $\quad 62 \mathrm{E} 15 \cdot 60 \mathrm{E} 05 \cdot 60 \mathrm{E} 10$

\section{Introduction}

Fisher (1915) derived the distribution of mean-centered sum of squares and sum of products in order to study the distribution of correlation coefficient from a bivariate normal sample. Let $X_{1}, X_{2}, \ldots, X_{N}(N>2)$ be two-dimensional independent random vectors where $X_{j}=\left(X_{1 j}, X_{2 j}\right)^{\prime}, j=1,2, \ldots, N$ is distributed as a bivariate normal distribution denoted by $N_{2}(\theta, \Sigma)$ with $\theta=\left(\theta_{1}, \theta_{2}\right)^{\prime}$ and a $2 \times 2$ covariance matrix $\Sigma=\left(\sigma_{i k}\right), i=1,2 ; k=1,2$. The sample mean-centered sums of squares and sum of products are given by $a_{i i}=\sum_{j=1}^{N}\left(X_{i j}-\bar{X}_{i}\right)^{2}=m S_{i}^{2}, m=N-1,(i=1,2)$ and $a_{12}=\sum_{j=1}^{N}\left(X_{1 j}-\bar{X}_{1}\right)\left(X_{2 j}-\bar{X}_{2}\right)=m R S_{1} S_{2}$, respectively. The quantity $R$

\footnotetext{
A. H. Joarder $(\bowtie)$

Department of Mathematics and Statistics, King Fahd University of Petroleum and Minerals, Dhahran 31261, Saudi Arabia

e-mail: anwarj@kfupm.edu.sa; ajstat@gmail.com
} 
is the sample product moment correlation coefficient. The distribution of $a_{11}, a_{22}$ and $a_{12}$ was derived by Fisher (1915) and may be called the bivariate Wishart distribution after Wishart (1928) who obtained the distribution of a $p$-variate Wishart matrix as the joint distribution of sample variances and covariances from the multivariate normal population. Obviously $a_{11} / \sigma_{11}$ has a chi-square distribution with $m$ degrees of freedom.

Let $U_{1}=m S_{1}^{2} / \sigma_{1}^{2}$ and $U_{2}=m S_{2}^{2} / \sigma_{2}^{2}$. The joint distribution of $U_{1}$ and $U_{2}$ is called the bivariate chi-square distribution after Krishnaiah et al. (1963). There are also a number of other bivariate chi-square and gamma distributions excellently reviewed by Kotz et al. (2000).

In this paper we derive its exact probability density function (pdf) in Theorem 2.1. Some properties of the distribution available in literature are reviewed in Kotz et al. (2000). We feel the properties need to be thoroughly investigated in the light of the joint pdf derived in this paper.

We provide moments of the product of two correlated chi-square random variables in Corollaries 3.2 and 3.3. In case the correlation coefficient vanishes, the moments, as expected, coincide with that of the bivariate independent chi-squares random variables. An application is pinpointed in connection with the distribution of sample product moment correlation coefficient based on a bivariate $t$ distribution. The bivariate $t$ distribution has fatter tails compared to bivariate normal distribution and has found numerous applications to business data especially stock return data.

Ratios of two independent chi-squares are widely used in statistical tests of hypotheses. However, testing with the ratio of correlated chi-squares variables are rarely noticed. If $\sigma_{1}=\sigma_{2}$, Finney (1938) derived the sampling distribution of the square root of the ratio of correlated chi-squares variables $(T=\sqrt{U / V})$ directly from the joint distribution of sample variances and correlation coefficient. He compared the variability of the measurements of standing height and stem length for different age groups of schoolboys by his method and by Hirsfeld (1937).

In Corollaries 3.7 and 3.8, we have provided moments of the ratio of two correlated chi-square variables. Further investigation is needed to utilize these moments to derive distributional properties of correlated chi-square variables by the inverse Mellin transform (Provost 1986). It needs further investigation to derive the marginal and conditional distribution, and the distribution of the sum, product, ratio and similar quantities directly from the joint density function of the bivariate chi-square distribution with pdf in Theorem 2.1.

In what follows, for any nonnegative integer $k$, we will use the notation

$$
c_{(k)}=c(c+1) \cdots(c+k-1), \quad c_{(0)}=1, \quad(c \neq 0) .
$$

\section{The pdf of the bivariate chi-square distribution}

The pdf of the bivariate Wishart distribution originally derived by Fisher (1915) can be written as 

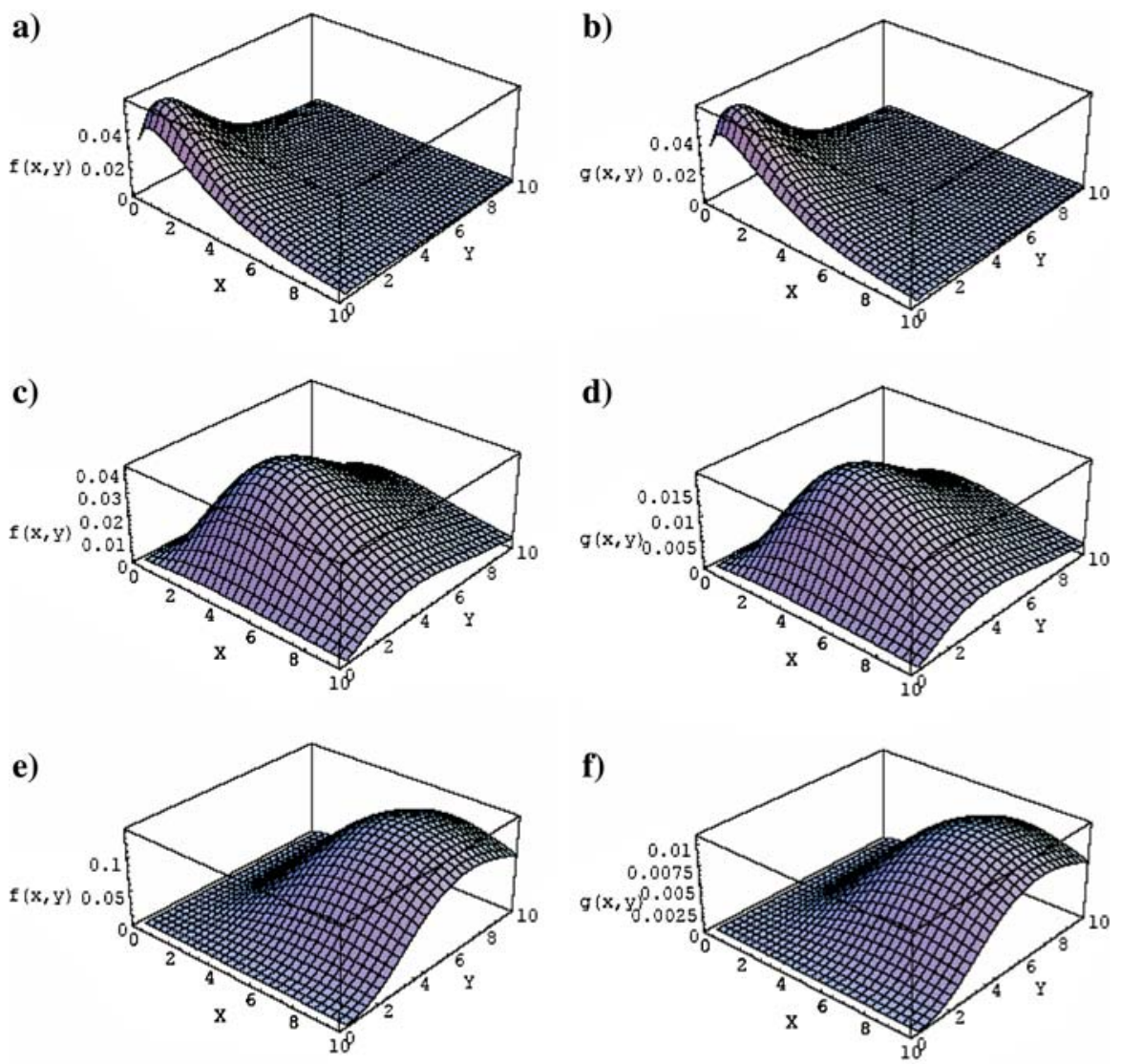

Fig. 1 Correlated bivariate chi-square density $f(x, y)$ surface with $\rho=0$ versus independent bivariate chi-square density $g(x, y)$ surfaces at $3(\mathbf{a}, \mathbf{b}), 6(\mathbf{c}, \mathbf{d})$, and $9(\mathbf{e}, \mathbf{f})$ degrees of freedom

$$
\begin{aligned}
f_{1}\left(a_{11}, a_{22}, a_{12}\right)= & \frac{\left(1-\rho^{2}\right)^{-m / 2}\left(\sigma_{1} \sigma_{2}\right)^{-m}}{2^{m} \sqrt{\pi} \Gamma\left(\frac{m}{2}\right) \Gamma\left(\frac{m-1}{2}\right)}\left(a_{11} a_{22}-a_{12}^{2}\right)^{(m-3) / 2} \\
& \times \exp \left(-\frac{a_{11}}{2\left(1-\rho^{2}\right) \sigma_{1}^{2}}-\frac{a_{22}}{2\left(1-\rho^{2}\right) \sigma_{2}^{2}}+\frac{\rho a_{12}}{\left(1-\rho^{2}\right) \sigma_{1} \sigma_{2}}\right)
\end{aligned}
$$

where $a_{11}>0, a_{22}>0,-\sqrt{a_{11} a_{22}}<a_{12}<\sqrt{a_{22} a_{22}}, m>2, \sigma_{1}^{2}=\sigma_{11}, \sigma_{2}^{2}=$ $\sigma_{22}, \sigma_{12}=\rho \sigma_{1} \sigma_{2}, \sigma_{1}>0, \sigma_{2}>0$ and $\rho,(-1<\rho<1)$, is the product moment correlation coefficient between $X_{1 j}$ and $X_{2 j}(j=1,2, \ldots, N)$ (Anderson 2003, p. 123) (Figs. 1, 2).

Since the correlation coefficient between $X_{1 j}$ and $X_{2 j}(j=1,2, \ldots, N)$ is $\rho$, the correlation between $U_{1}$ and $U_{2}$ is $\rho^{2}$ (Joarder 2006). In the following theorem we derive the pdf of the correlated bivariate chi-square distribution. 

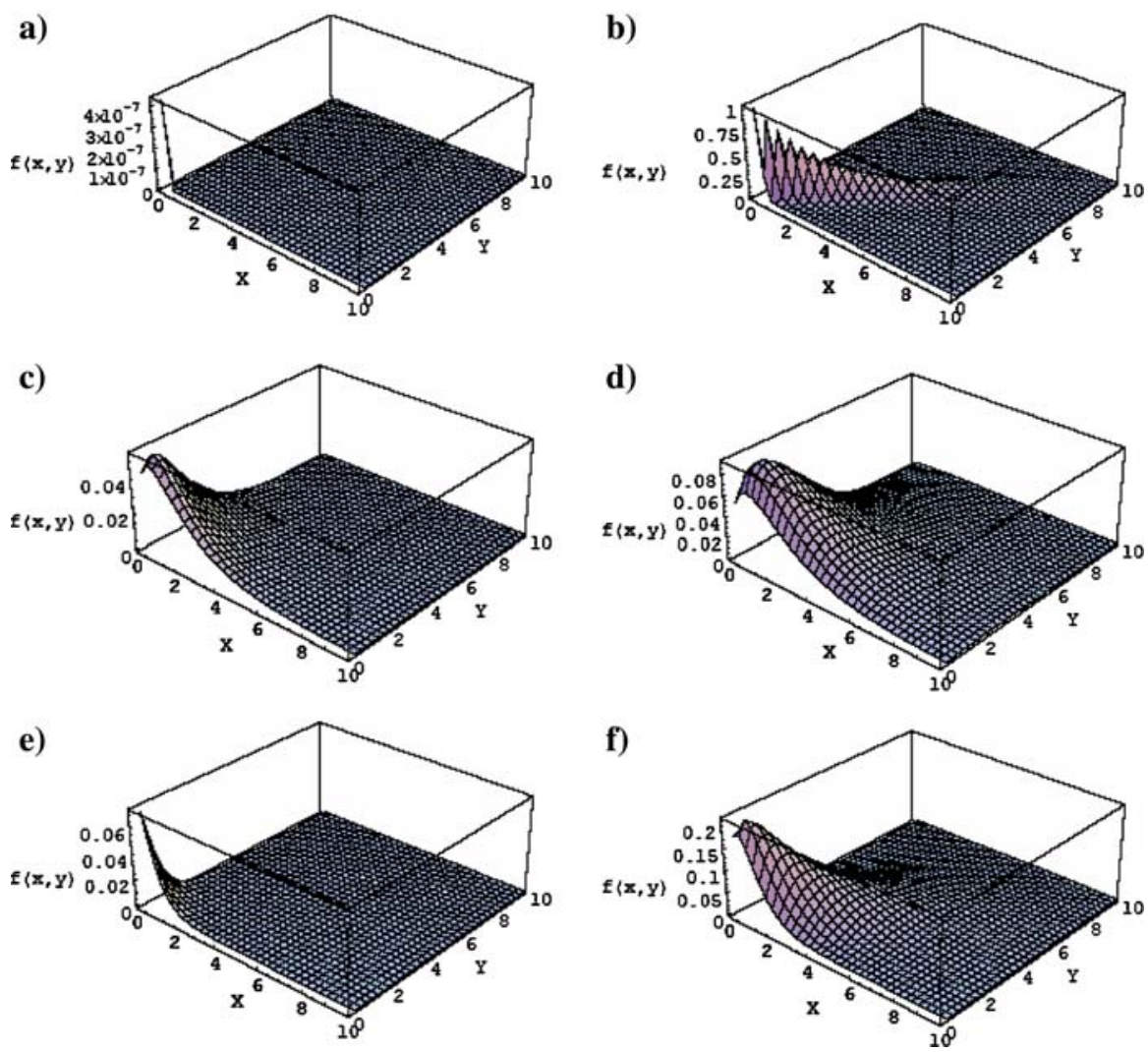

Fig. 2 Correlated bivariate chi-square density $f(x, y)$ surface with 3 degrees of freedom at different values of $\rho: \mathbf{a}(\rho=-0.99), \mathbf{b}(\rho=0.99), \mathbf{c}(\rho=-0.5), \mathbf{d}(\rho=0.5), \mathbf{e}(\rho=-0.85)$, and $\mathbf{f}(\rho=0.85)$

Theorem 2.1 The random variables $U_{1}$ and $U_{2}$ are said to have a correlated bivariate chi-square distribution each with $m$ degrees of freedom, if its $p d f$ is given by

$f\left(u_{1}, u_{2}\right)=\frac{2^{-(m+1)}\left(u_{1} u_{2}\right)^{(m-2) / 2} e^{\frac{-\left(u_{1}+u_{2}\right)}{2\left(1-\rho^{2}\right)}}}{\sqrt{\pi} \Gamma\left(\frac{m}{2}\right)\left(1-\rho^{2}\right)^{m / 2}} \sum_{k=0}^{\infty}\left[1+(-1)^{k}\right]\left(\frac{\rho \sqrt{u_{1} u_{2}}}{1-\rho^{2}}\right)^{k} \frac{\Gamma\left(\frac{k+1}{2}\right)}{k ! \Gamma\left(\frac{k+m}{2}\right)}$,

$m=N-1>2,-1<\rho<1$.

Proof Under the transformation $a_{11}=m s_{1}^{2}, a_{22}=m s_{2}^{2}, a_{12}=m r s_{1} s_{2}$ in (2.1) with Jacobian $J\left(\left(a_{11}, a_{22}, a_{12}\right) \rightarrow\left(s_{1}^{2}, s_{2}^{2}, r\right)\right)=m^{3} s_{1} s_{2}$, the pdf of $S_{1}^{2}, S_{2}^{2}$ and $R$ is given by

$$
\begin{aligned}
f_{2}\left(s_{1}^{2}, s_{2}^{2}, r\right)= & \left(\frac{m}{2 \sigma_{1} \sigma_{2}}\right)^{m} \frac{\left(1-\rho^{2}\right)^{-m / 2}}{\sqrt{\pi} \Gamma\left(\frac{m}{2}\right) \Gamma\left(\frac{m-1}{2}\right)}\left(1-r^{2}\right)^{(m-3) / 2}\left(s_{1} s_{2}\right)^{m-2} \\
& \times \exp \left(-\frac{m s_{1}^{2}}{2\left(1-\rho^{2}\right) \sigma_{1}^{2}}-\frac{m s_{2}^{2}}{2\left(1-\rho^{2}\right) \sigma_{2}^{2}}+\frac{m \rho r s_{1} s_{2}}{\left(1-\rho^{2}\right) \sigma_{1} \sigma_{2}}\right) .
\end{aligned}
$$


By making the transformation $m s_{1}^{2}=\sigma_{1}^{2} u_{1}, m s_{2}^{2}=\sigma_{2}^{2} u_{2}$, keeping $r$ intact, with Jacobian $J\left(s_{1}^{2}, s_{2}^{2} \rightarrow u_{1} u_{2}\right)=\left(\sigma_{1} \sigma_{2} / m\right)^{2}$, we have

$$
\begin{aligned}
f_{3}\left(u_{1}, u_{2}, r\right)= & \frac{\left(1-\rho^{2}\right)^{-m / 2}\left(u_{1} u_{2}\right)^{(m-2) / 2}\left(1-r^{2}\right)^{(m-3) / 2}}{2^{m} \sqrt{\pi} \Gamma\left(\frac{m}{2}\right) \Gamma\left(\frac{m-1}{2}\right)} \\
& \times \exp \left(-\frac{u_{1}+u_{2}}{2\left(1-\rho^{2}\right)}+\frac{\rho r \sqrt{u_{1} u_{2}}}{1-\rho^{2}}\right) .
\end{aligned}
$$

Then integrating out $r$, the joint pdf of $U_{1}$ and $U_{2}$ is given by

$$
\begin{aligned}
f_{3}\left(u_{1}, u_{2}\right)= & \frac{\left(1-\rho^{2}\right)^{-m / 2}\left(u_{1} u_{2}\right)^{(m-2) / 2}}{2^{m} \sqrt{\pi} \Gamma\left(\frac{m}{2}\right) \Gamma\left(\frac{m-1}{2}\right)} \exp \left(-\frac{u_{1}+u_{2}}{2\left(1-\rho^{2}\right)}\right) \\
& \times \int_{-1}^{1}\left(1-r^{2}\right)^{(m-3) / 2} \exp \left(\frac{\rho r \sqrt{u_{1} u_{2}}}{1-\rho^{2}}\right) d r .
\end{aligned}
$$

By expanding the last exponential term of the above density function we have

$$
\begin{aligned}
f_{3}\left(u_{1}, u_{2}\right)= & \frac{\left(1-\rho^{2}\right)^{-m / 2}\left(u_{1} u_{2}\right)^{(m-2) / 2}}{2^{m} \sqrt{\pi} \Gamma\left(\frac{m}{2}\right) \Gamma\left(\frac{m-1}{2}\right)} \exp \left(-\frac{u_{1}+u_{2}}{2\left(1-\rho^{2}\right)}\right) \\
& \times \sum_{k=0}^{\infty} \frac{1}{k !}\left(\frac{\rho r \sqrt{u_{1} u_{2}}}{1-\rho^{2}}\right)^{k}\left[\int_{-1}^{0} r^{k}\left(1-r^{2}\right)^{(m-3) / 2}+\int_{0}^{1} r^{k}\left(1-r^{2}\right)^{(m-3) / 2}\right] d r
\end{aligned}
$$

which can be written as

$$
\begin{aligned}
f_{3}\left(u_{1}, u_{2}\right)= & \frac{\left(1-\rho^{2}\right)^{-m / 2}\left(u_{1} u_{2}\right)^{(m-2) / 2}}{2^{m} \sqrt{\pi} \Gamma\left(\frac{m}{2}\right) \Gamma\left(\frac{m-1}{2}\right)} \exp \left(-\frac{u_{1}+u_{2}}{2\left(1-\rho^{2}\right)}\right) \\
& \times \sum_{k=0}^{\infty}\left[(-1)^{k}+1\right]\left(\frac{\rho \sqrt{u_{1} u_{2} y}}{1-\rho^{2}}\right)^{k} \frac{1}{k !} \int_{0}^{1}(1-y)^{(m-3) / 2}\left(\frac{1}{2} y^{-1 / 2}\right) d y .
\end{aligned}
$$

By completing the beta integral of the above density function, we have

$$
\begin{aligned}
f_{3}\left(u_{1}, u_{2}\right)= & \frac{\left(1-\rho^{2}\right)^{-m / 2}\left(u_{1} u_{2}\right)^{(m-2) / 2}}{2^{m+1} \sqrt{\pi} \Gamma\left(\frac{m}{2}\right) \Gamma\left(\frac{m-1}{2}\right)} \exp \left(-\frac{u_{1}+u_{2}}{2\left(1-\rho^{2}\right)}\right) \\
& \times \sum_{k=0}^{\infty}\left[1+(-1)^{k}\right]\left(\frac{\rho \sqrt{u_{1} u_{2} y}}{1-\rho^{2}}\right)^{k} \frac{1}{k !} \frac{\Gamma\left(\frac{k+1}{2}\right) \Gamma\left(\frac{m-1}{2}\right)}{\Gamma\left(\frac{k+m}{2}\right)}
\end{aligned}
$$

which simplifies to what we have the theorem. 
Note that the pdf of the correlated bivariate chi-square distribution each with $m$ degrees of freedom can be written as

$$
f\left(u_{1}, u_{2}\right)=\frac{\left(u_{1} u_{2}\right)^{(m-2) / 2} e^{\frac{-\left(u_{1}+u_{2}\right)}{2\left(1-\rho^{2}\right)}}}{2^{m} \sqrt{\pi} \Gamma\left(\frac{m}{2}\right)\left(1-\rho^{2}\right)^{m / 2}} \sum_{k=0}^{\infty}\left(\frac{\rho \sqrt{u_{1} u_{2}}}{1-\rho^{2}}\right)^{k} \frac{\Gamma\left(\frac{k+1}{2}\right)}{k ! \Gamma\left(\frac{k+m}{2}\right)},
$$

$m=N-1>2,-1<\rho<1$, and $k=0,2,4, \ldots$

In case $\rho=0$, the pdf in Theorem 2.1 becomes the product of that of the two independent chi-square random variables each with $m$ degrees of freedom.

\section{Moments of the product and ratio of two correlated chi-square random variables}

Theorem 3.1 The $(a, b)$-th product moment $\mu^{\prime}(a, b ; \rho)=E\left(U_{1}^{a} U_{2}^{b}\right)$ is given by

$$
\begin{aligned}
E\left(U_{1}^{a} U_{2}^{b}\right)= & \frac{2^{a+b-1}\left(1-\rho^{2}\right)^{a+b}}{L(m, \rho)} \\
& \times \sum_{k=0}^{\infty}\left[1+(-1)^{k}\right] \frac{(2 \rho)^{k}}{k ! \Gamma\left(\frac{k+m}{2}\right)} \Gamma\left(\frac{k+m}{2}+a\right) \Gamma\left(\frac{k+m}{2}+b\right) \Gamma\left(\frac{k+1}{2}\right)
\end{aligned}
$$

where $m>2 \max (a, b),-1<\rho<1$ and

$$
L(m, \rho)=\sqrt{\pi} \Gamma(m / 2)\left(1-\rho^{2}\right)^{-m / 2} .
$$

Proof It follows from Theorem 2.1 that the $(a, b)$-th product moment of the distribution of $U_{1}$ and $U_{2}$ is given by

$$
\begin{aligned}
E\left(U_{1}^{a} U_{2}^{b}\right)= & \frac{\left(1-\rho^{2}\right)^{-m / 2}}{2^{m} \sqrt{\pi} \Gamma\left(\frac{m}{2}\right)} \\
& \times \sum_{k=0}^{\infty}\left[1+(-1)^{k}\right]\left(\frac{\rho}{1-\rho^{2}}\right)^{k} \frac{1}{k !} \frac{\Gamma\left(\frac{k+1}{2}\right)}{\Gamma\left(\frac{k+m}{2}\right)} \\
& \times \int_{0}^{\infty} \int_{0}^{\infty} u_{1}^{\frac{m+k}{2}+a-1} u_{2}^{\frac{m+k}{2}+b-1} e^{-\frac{\left(u_{1}+u_{2}\right)}{2\left(1-\rho^{2}\right)}} d u_{1} d u_{2} .
\end{aligned}
$$

The theorem then follows by having evaluated the above integrals.

Obviously $\mu^{\prime}(a, b ; \rho)=\mu^{\prime}(b, a ; \rho)=E\left(U_{1}^{a} U_{2}^{b}\right)$. If $\rho=0$, the product moment in Theorem 3.1 simplifies to

$$
E\left(U_{1}^{a} U_{2}^{b}\right)=\frac{2^{a+b}}{\Gamma^{2}(m / 2)} \Gamma\left(\frac{m}{2}+a\right) \Gamma\left(\frac{m}{2}+b\right)
$$


which is the product of the $a$-th and $b$-th moments of two independent variables $U_{1} \sim \chi_{m}^{2}$ and $U_{2} \sim \chi_{m}^{2}$. In case $a$ is a nonnegative integer, then

$$
\Gamma\left(\frac{k+m}{2}+a\right)=\Gamma\left(\frac{k+m}{2}\right)\left(\frac{k+m}{2}\right)_{(a)} .
$$

Corollary 3.1 If the integers (i) $a$ and $b$ are nonnegative, or, (ii) $a>0, b<0$ with $m>2 b$, then the product moment in Theorem 3.1 can be written as

$$
E\left(U_{1}^{a} U_{2}^{b}\right)=\frac{2^{a+b}\left(1-\rho^{2}\right)^{a+b+m / 2}}{\sqrt{\pi} \Gamma\left(\frac{m}{2}\right)} \sum_{k=0}^{\infty} \rho^{k} \gamma_{k, m+2 b}\left(\frac{k+m}{2}\right)_{(a)}
$$

where

$$
\gamma_{k, m}=\left[1+(-1)^{k}\right] \frac{2^{k-1}}{k !} \Gamma\left(\frac{k+1}{2}\right) \Gamma\left(\frac{k+m}{2}\right) .
$$

Plugging in $a=b$ in Corollary 3.1, we have the following corollary.

Corollary 3.2 For $m>2,-1<\rho<1$, the a-th moment of $V=U_{1} U_{2}$ is given by

$$
E\left(V^{a}\right)=\frac{4^{a}\left(1-\rho^{2}\right)^{2 a+m / 2}}{\sqrt{\pi} \Gamma\left(\frac{m}{2}\right)} \sum_{k=0}^{\infty} \frac{\Gamma\left(\frac{k+m}{2}+a\right)}{\Gamma\left(\frac{k+m}{2}\right)} \gamma_{k, m+2 a} \rho^{k}
$$

where $\gamma_{k, m}$ is defined by (3.4).

In case $a$ is a nonnegative integer, then by the use of (3.2) in (3.5) we have the following:

$$
E\left(V^{a}\right)=\frac{4^{a}\left(1-\rho^{2}\right)^{2 a+m / 2}}{\sqrt{\pi} \Gamma\left(\frac{m}{2}\right)} \sum_{k=0}^{\infty}\left(\frac{k+m}{2}\right)_{(a)} \gamma_{k, m+2 a} \rho^{k}
$$

where $\gamma_{k, m}$ is defined by (3.4). The moment generating function of $V$ at $h$ is given by

$$
M_{V}(h)=E\left(e^{h V}\right)=E \sum_{a=0}^{\infty} \frac{(h V)^{a}}{a !}=\sum_{a=0}^{\infty} \frac{h^{a}}{a !} E\left(V^{a}\right) .
$$

The moments of $V$ are evaluated from Corollary 3.2.

Corollary 3.3 For $m>2,-1<\rho<1$, the first four raw moments of Vare given by

(i) $E(V)=m\left(m+2 \rho^{2}\right)$,

(ii) $E\left(V^{2}\right)=m(m+2)\left[8 \rho^{4}+8(m+2) \rho^{2}+m(m+2)\right]$,

(iii) $E\left(V^{3}\right)=16\left(\frac{m}{2}\right)_{(3)}\left[24 \rho^{6}+36(m+4) \rho^{4}+36\left(\frac{m}{2}+1\right)_{(2)} \rho^{2}+4\left(\frac{m}{2}\right)_{(3)}\right]$, 
(iv) $E\left(V^{4}\right)=256\left(\frac{m}{2}\right)_{(4)}\left[24 \rho^{8}+48(m+6) \rho^{6}+72\left(\frac{m}{2}+2\right)_{(2)} \rho^{4}\right.$

$$
\left.+16\left(\frac{m}{2}+1\right)_{(3)} \rho^{2}+\left(\frac{m}{2}\right)_{(4)}\right] \text {. }
$$

Proof Define $L^{(r)}(m, \rho)=\sum_{k=0}^{\infty} k^{(r)} \gamma_{k, m} \rho^{k}$, with $L^{(0)}(m, \rho)=\sum_{k=0}^{\infty} \gamma_{k, m} \rho^{k}$ which is denoted by $L(m, \rho)$ (Joarder 2006), and given in (3.1) in this paper. Then it can be checked that $L^{(1)}(m+2, \rho)=(m+2) \rho^{2}\left(1-\rho^{2}\right) L(m+2, \rho)$, and that

$$
\begin{aligned}
\sum_{k=0}^{\infty} \rho^{k} \gamma_{k, m+2}((k+m) / 2)= & \frac{1}{2}\left[L^{(1)}(m+2, \rho)+m L(m+2, \rho)\right] \\
= & \frac{1}{2}\left[(m+2) \rho^{2}\left(1-\rho^{2}\right)^{-1}+m\right] L(m+2, \rho) \\
= & \frac{\sqrt{\pi}}{2} \Gamma\left(\frac{m}{2}+1\right)\left[(m+2) \rho^{2}\left(1-\rho^{2}\right)^{-1}+m\right] \\
& \times\left(1-\rho^{2}\right)^{-(m+2) / 2} .
\end{aligned}
$$

By plugging the above in to (3.6) with $a=1$, we have $E(V)=\left(1-\rho^{2}\right)[m+$ $\left.(m+2) \rho^{2}\left(1-\rho^{2}\right)\right]$ which simplifies to (i).

(ii) Using the following identities

$$
\begin{aligned}
& L^{(1)}(m+4, \rho)=(m+4) \rho^{2}\left(1-\rho^{2}\right) L(m+4, \rho) \quad \text { and } \\
& L^{(2)}(m+4, \rho)=\left[(m+4)(m+5) \rho^{4}+(m+4) \rho^{2}\right]\left(1-\rho^{2}\right)^{-2} L(m+4, \rho)
\end{aligned}
$$

in the expression

$$
\begin{aligned}
& \sum_{k=0}^{\infty} \rho^{k} \gamma_{k, m+4}((k+m) / 2)_{(2)} \\
& \quad=\frac{1}{4}\left[L^{(2)}(m+4, \rho)+(2 m+3) L^{(1)}(m+4, \rho)+m(m+2) L(m+4, \rho)\right]
\end{aligned}
$$

and plugging in the value of $L(m+4, \rho)$ in (3.6) with $a=2$, we get (ii).

Similarly (iii) and (iv) can be proved.

In has been mentioned at the end of Theorem 2.1 that if $\rho=0$, then $U_{1} \sim \chi_{m}^{2}$ and $U_{2} \sim \chi_{m}^{2}$ will be independent. In that case, $V$ will be the product of two independent chi-square random variables each with $m$ degrees of freedom and evidently the resulting moments will be in agreement with that situation. For example if $\rho=0$, from Corollary 3.3 (iv) we have $E\left(V^{4}\right)=[m(m+2)(m+4)(m+6)]^{2}$ which is the product of the fourth moments of two independent chi-squares each having $m$ degrees of freedom, i.e., $E\left(V^{4}\right)=E\left(U_{1}^{4}\right) E\left(U_{2}^{4}\right)$.

Since the geometric mean of the chi-square variables are important in many applications, we define $G=\sqrt{U_{1} U_{2}}$, the geometric mean of $U_{1}$ and $U_{2}$, and provide its moments below. 
Corollary 3.4 For $m>2,-1<\rho<1$, the first four raw moments of $G$ are given by

(i) $E(G)=\frac{2\left(1-\rho^{2}\right)^{(m+2) / 2}}{\sqrt{\pi} \Gamma\left(\frac{m}{2}\right)} \sum_{k=0}^{\infty} \frac{\Gamma\left(\frac{k+m+1}{2}\right)}{\Gamma\left(\frac{k+m}{2}\right)} \gamma_{k, m+1} \rho^{k}$,

(ii) $E\left(G^{2}\right)=m\left(m+2 \rho^{2}\right)$,

(iii) $E\left(G^{3}\right)=\frac{8\left(1-\rho^{2}\right)^{(m+6) / 2}}{\sqrt{\pi} \Gamma\left(\frac{m}{2}\right)} \sum_{k=0}^{\infty} \frac{\Gamma\left(\frac{k+m+3}{2}\right)}{\Gamma\left(\frac{k+m}{2}\right)} \gamma_{k, m+3} \rho^{k}$,

(iv) $E\left(G^{4}\right)=m(m+2)\left[8 \rho^{4}+8(m+2) \rho^{2}+m(m+2)\right]$.

Since $G=\sqrt{U_{1} U_{2}}=\sqrt{V}$, the moment generating function of $G$ at $h$ is given by $E\left(e^{h G}\right)=\sum_{a=0}^{\infty} \frac{h^{a}}{a !} E\left(G^{a}\right)=\sum_{a=0}^{\infty} \frac{h^{a}}{a !} E\left(V^{a / 2}\right)$. Then we have the following corollary.

Corollary 3.5 If a is nonnegative with $m>2 a$, then for $-1<\rho<1$, the moment generating function of $G$ at $h$ is given by

$$
M_{G}(h)=\frac{\left(1-\rho^{2}\right)^{m / 2}}{\sqrt{\pi} \Gamma\left(\frac{m}{2}\right)} \sum_{a=0}^{\infty} \frac{(2 h)^{a}\left(1-\rho^{2}\right)^{a}}{a !} \sum_{k=0}^{\infty} \frac{\Gamma\left(\frac{k+m+a}{2}\right)}{\Gamma\left(\frac{k+m}{2}\right)} \gamma_{k, m+a} \rho^{k},
$$

where $\gamma_{k, m}$ is defined by (3.4). The following results from Corollaries 3.2 and 3.5 by putting $\rho=0$.

Corollary 3.6 Let $G=\sqrt{U_{1} U_{2}}=\sqrt{V}$ be the geometric mean of two independent chi-square random variables $U_{1}$ and $U_{2}$ each with $m$ degrees of freedom. Then the $a$-th moment and the moment generating function of $G$ at $h$ are given by

$$
\begin{aligned}
& E\left(G^{a}\right)=\frac{2^{a} \Gamma^{2}\left(\frac{m+a}{2}\right)}{\Gamma^{2}\left(\frac{m}{2}\right)} \text { and } \\
& M_{G}(h)=\sum_{a=0}^{\infty} \frac{(2 h)^{a}}{a !} \frac{\Gamma^{2}\left(\frac{m+a}{2}\right)}{\Gamma^{2}\left(\frac{m}{2}\right)},
\end{aligned}
$$

respectively (Joarder 2007).

Let $W=U_{1} / U_{2}$, the ratio of two correlated chi-square variables $U_{1}$ and $U_{2}$ that have pdf in Theorem 2.1. Then the following result follows from Theorem 3.1.

Corollary 3.7 If $a$ is nonnegative with $m>2 a$, then for $-1<\rho<1$, the $a$-th moment of $W$ is given by

$$
E\left(W^{a}\right)=\frac{\left(1-\rho^{2}\right)^{m / 2}}{\sqrt{\pi} \Gamma(m / 2)} \sum_{k=0}^{\infty} \frac{\Gamma\left(\frac{k+m}{2}+a\right)}{\Gamma\left(\frac{k+m}{2}\right)} \gamma_{k, m-2 a} \rho^{k},
$$

where $\gamma_{k, m-2 a}$ is defined by (3.4). In case a is a nonnegative integer, we have

$$
E\left(W^{a}\right)=\frac{\left(1-\rho^{2}\right)^{m / 2}}{\sqrt{\pi} \Gamma\left(\frac{m}{2}\right)} \sum_{k=0}^{\infty}\left(\frac{k+m}{2}\right)_{(a)} \gamma_{k, m-2 a} \rho^{k}, \quad m>2 a, \quad-1<\rho<1 .
$$


The moments of $W$ up to fourth order are calculated below from Corollary 3.7. The derivation is similar to that of Corollary 3.3.

Corollary 3.8 For $m>8,-1<\rho<1$, the first four moments of $W$ are given below:

(i) $E(W)=\frac{m-2 \rho^{2}}{m-2}, m>2$,

(ii) $E\left(W^{2}\right)=\frac{1}{(m-2)(m-4)}\left(24 \rho^{4}-8(m+2) \rho^{2}+m(m+2)\right), m>4$,

(iii) $E\left(W^{3}\right)=\frac{\Gamma\left(\frac{m}{2}-3\right)}{8 \Gamma\left(\frac{m}{2}\right)}\left[-480 \rho^{6}+144(m+4) \rho^{4}-72\left(\frac{m}{2}+1\right)_{(2)} \rho^{2}+8\left(\frac{m}{2}\right)_{(3)}\right]$, $m>6$,

(iv) $E\left(W^{4}\right)=\frac{\Gamma\left(\frac{m}{2}-4\right)}{16 \Gamma\left(\frac{m}{2}\right)}\left[13440 \rho^{8}-3840(m+6) \rho^{6}+1920\left(\frac{m}{2}+2\right){ }_{(2)} \rho^{4}\right.$ $\left.-256\left(\frac{m}{2}+1\right)_{(3)} \rho^{2}+8\left(\frac{m}{2}\right)_{(3)}\right], m>8$.

In case $\rho=0$, then $W$ will be the ratio of two independent chi-square variables each with the same degrees of freedom, and the above moments will be simply

$$
E\left(W^{a}\right)=\frac{\left(\frac{m}{2}\right)_{(a)}}{\left(\frac{m}{2}-a\right)_{(a)}}, \quad m>2 a
$$

which are evidently in agreement with the resulting situation of independence.

Corollary 3.9 If $a$ is an integer with $m>2 a$, then for $-1<\rho<1$, the moment generating function of $W$ at $h$ is given by

$$
M_{W}(h)=\frac{\left(1-\rho^{2}\right)^{m / 2}}{\sqrt{\pi} \Gamma\left(\frac{m}{2}\right)} \sum_{a=0}^{\infty} \frac{h^{a}}{a !} \sum_{k=0}^{\infty}\left(\frac{k+m}{2}\right)_{(a)} \gamma_{k, m-2 a} \rho^{k}
$$

where $L(m, \rho)$ is defined in Theorem 3.1.

\section{The distribution of the correlation coefficient based on bivariate $t$ population}

The lengthy proof of the distribution of sample correlation coefficient based on a bivariate normal distribution by Fisher (1915) has been made shorter and elegant by Joarder (2007). Further he derived the distribution of correlation coefficient for bivariate $t$ distribution along Fisher (1915).

It is known that the distribution of correlation coefficient is robust under violation of normality at least in the bivariate elliptical class of distributions (Fang and Anderson 1990, p. 10; Fang and Zhang 1990, p. 137, or Ali and Joarder 1991). However a direct derivation by Joarder (2007) provides more insight for those who have been recently using $t$ distributions as parent populations. 
Theorem 4.1 The pdf of the correlation coefficient $R$ based on a bivariate t population is given by

(i) $h(r)=\frac{2^{m-2} \Gamma^{2}\left(\frac{m}{2}\right)\left(1-\rho^{2}\right)^{m / 2}}{\pi \Gamma(m-1)}\left(1-r^{2}\right)^{(m-3) / 2} M_{G}(\rho r)$

(ii) $h(r)=\frac{2^{m-2}\left(1-\rho^{2}\right)^{m / 2}}{\pi \Gamma(m-1)}\left(1-r^{2}\right)^{(m-3) / 2} \sum_{k=0}^{\infty} \frac{(2 \rho r)^{k}}{k !} \Gamma^{2}\left(\frac{m+k}{2}\right)$,

$-1<r<1$ (cf. Muirhead 1982, p. 154) where $m>2,-1<\rho<1$ and $M_{G}(\rho r)$ is defined in Corollary 3.6.

It is overviewed here to show the application of the moments of Sect. 3. Since part (ii) of Theorem 4.1 is well known to be the distribution of the correlation coefficient for bivariate normal population, it proves the robustness of the distribution or of tests on correlation coefficient. Thus the assumption of bivariate normality under which many tests on correlation coefficient are developed can be relaxed to a broader class of bivariate $t$ distribution. Interested readers may go through Muddapur (1988) for some exciting exact tests on correlation coefficient. Further the readers are referred to Joarder and Ahmed (1996), Billah and Saleh (2000), Kibria (2004), Kibria and Saleh (2004), Kotz and Nadarajah (2004) and the references therein for applications of $t$ distribution.

Acknowledgments The author acknowledges the excellent research support provided by King Fahd University of Petroleum and Minerals, Saudi Arabia through the Project FT 2004-2. The author also thanks the referees, his colleague Dr. M.H. Omar, and Professor M. Ismail, University of Central Florida, for constructive suggestions that have improved the original version in many ways.

Open Access This article is distributed under the terms of the Creative Commons Attribution Noncommercial License which permits any noncommercial use, distribution, and reproduction in any medium, provided the original author(s) and source are credited.

\section{References}

Ali MM, Joarder AH (1991) Distribution of the correlation coefficient for the class of bivariate elliptical models. Can J Stat 19:447-452

Anderson TW (2003) An introduction to multivariate statistical analysis. Wiley, London

Billah MB, Saleh AKME (2000) Performance of the large sample tests in the actual forecasting of the pretest estimators for regression model with $t$ error. J Appl Stat Sci 9(3):237-251

Fang KT, Anderson TW (1990) Statistical inference in elliptically contoured and related distributions. Allerton Press

Fang KT, Zhang YT (1990) Generalized multivariate analysis. Springer, Heidelberg

Finney DJ (1938) The distribution of the ratio of the estimates of the two variances in a sample from a bivariate normal population. Biometrika 30:190-192

Fisher RA (1915) Frequency distribution of the values of the correlation coefficient in samples from an indefinitely large population. Biometrika 10:507-521

Hirsfeld HO (1937) The distribution of the ratio of covariance estimates in two samples drawn from normal bivariate populations. Biometrika 29:65-79

Joarder AH (2006) Product moments of bivariate Wishart distribution. J Probab Stat Sci 4(2):233-243

Joarder AH (2007) Some useful integrals and their applications in correlation analysis. Stat Papers (in press) Joarder AH, Ahmed SE (1996) Estimation of characteristic roots of scale matrix. Metrika 4:59-67

Kibria BMG (2004) Conflict among the shrinkage estimators induced by W, LR and LM tests under a Student's t regression model. J Korean Stat Soc 33(4):411-433

Kibria BMG, Saleh AKME (2004) Preliminary test ridge regression estimators with Students' t errors and conflicting test statistics. Metrika 59(2):105-124 
Kotz S, Nadarajah S (2004) Multivariate $t$ distributions and their applications. Cambridge University Press, London

Kotz S, Balakrishnan N, Johnson NL (2000) Continuous multivariate distributions (vol 1: models and applications). Wiley, New York

Krishnaiah PR, Hagis P, Steinberg L (1963) A note on the bivariate chi-distribution. SIAM Rev 5:140-144

Muddapur MV (1968) A simple test for correlation coefficient in a bivariate normal distribution. Sankhya Ser B 50:50-68

Muirhead RJ (1982) Aspects of multivariate statistical theory. Wiley, New York

Provost SB (1986) The exact distribution of the ratio of a linear combination of chi-square variables over the root of a product of chi-square variables. Can J Stat 14(1):61-67

Wishart J (1928) The generalized product moment distribution in samples from a normal multivariate population. Biometrika A20:32-52 